\title{
Management of dysthyroid eye disease
}

\section{Surgical decompression}

The pathogenesis of dysthyroid eye disease continues to be an enigma though immune mechanisms with infiltration of the extraocular muscles by $T$ cells are certainly involved. The patient may suffer visual loss from optic nerve compression at the orbital apex or, in cases of gross proptosis with chemosis and failure of the lids to close, corneal drying, ulceration, and endophthalmitis. Both of these conditions require urgent intervention.

If there is endophthalmitis with severe proptosis and chemosis, then surgical orbital decompression should be carried out as soon as possible. The only reason for any brief delay would be to bring concomitant hyperthyroidism under control to avoid the risk of thyroid crisis reaction. Decompression $^{1}$ may be via a Caldwell-Luc approach, allowing decompression into the ethmoid space and the antrum on both the medial and if necessary lateral sides of the infraorbital nerve. Other surgeons prefer the translid approach, in which the incision through the nasal and medial parts of the lower lid gives good access to the same ethmoidal and antral spaces from the orbital side. Where maximal decompression is required, some surgeons add a third wall, namely the lateral wall, to the decompression, but it is rarely necessary to include the orbital roof as well, with a significant rise in morbidity. Once the necessary space has been opened by removal of bone, the periosteum should be incised from posterior to anterior with longitudinally running incisions to allow the tense orbital fat to herniate outwards. ${ }^{2}$ To reduce the risk of producing an A-pattern of esotropia, which commonly follows successful orbital decompression, Koornneef $^{2}$ has recommended that in the anterior part of the orbit the periosteal incisions run circumferentially rather than postero-anteriorly to minimise the damage to the complex orbital connective tissue framework which is essential for normal ocular movements. Unless the ophthalmologist is fully familiar with orbital and sinus anatomy it is usually preferable to carry out such surgery together with his ear, nose, and throat colleague so that he does the bone work and the eye surgeon carries out the periorbital incisions and the last stage of the decompression.

When optic nerve function is threatened by the enlarged ocular muscles compressing the nerve at the orbital apex, it is essential that the decompression goes back as far as possible on the medial side of the orbit to relieve the pressure. Although this may be achieved by either of the two routes mentioned above, I prefer the direct anterior ethmoidectomy route via an incision along the lateral side of the nose, which gives best access to the posterior ethmoidal area practically down to the optic foramen.

Some surgeons carry out orbital decompression for purely cosmetic reasons, and the Tessier approach, ${ }^{3}$ turning down a large frontal skin flap from behind the hairline, gives good access to all four orbital walls, and the periorbita can then be incised in all four quadrants to give excellent decompression with minimal risk of altering the ocular movements. The operation takes a long time. ophthalmologists. Others prefer to consider medical decompression, using high-dose oral prednisolone, $80-100 \mathrm{mg}$ daily, provided that there is no contraindication such as previous tuberculosis or peptic ulceration. The addition of cyclosporin to prednisolone is also effective; it permits a lower total dose of prednisolone and is more tolerable to the patient. Recently intensive intravenous methyl prednisolone $(0.5 \mathrm{~g}$ in $200 \mathrm{ml}$ of isotonic saline over 30 minutes, which can be repeated after 48 hours) has proved to be effective, but careful medical monitoring is required because of the cardiovascular risks. ${ }^{4}$ If patients having corticosteroid therapy are not showing signs of improvement in visual acuity after 48 hours, it may still be necessary to change to surgical decompression. Even when the patient does respond well to the steroid treatment, it is not always easy to reduce the dosage over the next few weeks to low levels without the risk of recurrence of symptoms of optic neuropathy. If the maintenance dose has to be $25 \mathrm{mg}$ of prednisolone or higher each day, many patients will not accept the frequent side effects that may accompany such levels.

\section{Radiotherapy}

Recently a third method of decompression has come back into vogue, namely radiotherapy, ${ }^{56}$ because improvements in the technique of administration together with the use of megavoltages from a linear accelerator are giving good results. Analysis of the results of such therapy in 311 patients treated over 20 years at Stanford showed improvement in soft tissue involvement, in proptosis, and in optic neuropathy. Male patients still requiring treatment for hyperthyroidism responded less favourably. The patients were treated with $2000 \mathrm{cGy}$ in 10 fractions in the majority of cases. Those treated with $3000 \mathrm{cGy}$ in 15 fractions of $200 \mathrm{cGy}$ did not suffer additional complications but neither did they have any improved outcome. Of these patients $29 \%$ had ocular surgery following the radiotherapy, and in most cases this was for diplopia with excellent results. Fears that the radiotherapy would increase orbital fibrosis and diplopia were not realised.

\section{New tests}

Pain, tenderness, and increased conjunctival injection are being used as indicators of active inflammation ${ }^{7}$ within the orbit, and the proposal is that at such time immunosuppression by either radiotherapy or corticosteroids would have the best opportunity of being effective. Methods of detecting early dysthyroid optic neuropathy have included the pattern electroretinogram (PERG) and cortical visual evoked potentials (CVEPs), but neither test is as useful as colour contrast sensitivity along the tritan (blue) axis. ${ }^{8}$ The tritan sensitivity losses increase in magnitude progressively in line with increasing severity of dysthyroid optic neuropathy as estimated by the usual clinical indicators. These tests may help in identifying the most useful time at which to attempt more active intervention.
Medical decompression

Surgical decompression may be the first choice for some
Diplopia

One of the more debilitating symptoms in this condition is 
double vision. Teaching in the past used to recommend that the ocular movements must have been shown to be stable for at least two years before ocular muscle surgery could be contemplated. This waiting time has now been reduced to six months of demonstrable stability by means of serial Hess charts and records of the field of binocular single vision. Rather surprisingly, botulinum neurotoxin A injections into the tight rectus muscles within one year of the onset of diplopia have sometimes given improved ocular rotations lasting much longer than the normal two months after such an injection. ${ }^{9}$ In seven out of 43 patients a long-lasting and stable result followed the use of one or two injections only. Since botulinum toxin cannot have a lytic effect on established fibrosis, it must be working in some other manner. In five patients with ophthalmic Graves' disease of recent onset length-tension measurements during surgery ${ }^{10}$ under topical anaesthesia suggested that the restricted ocular movements were caused by active muscle contraction in the early phase of the disease and not by fibrosis.

\section{Prospects}

Measuring blue contrast sensitivity, and recognising clinically the periods of active inflammation, will enable treatments with radiotherapy, corticosteroids, or botulinum toxin A to be given at the optimal time. Eventually the immunopathogenic mechanisms will be identified and stopped before the disease process becomes established.

Moorfields Eye Hospital,

PETER FELLS

London

1 Fells P. Orbital decompression for severe dysthyroid eye disease. $\mathrm{Br} \mathcal{J}$ Ophthalmol 1987; 71: 107-11.

2 Koornneef L. Eyelid and orbital fascial attachments and their clinical significance. Eye 1988; 2: 130-4.

3 Mourits MPh, Koornneef L, Wiersinga WM, Prummel MF, Berghout A, van der Gaag R. Orbital decompression for Graves' ophthalmopathy by inferomedial, by inferomedial plus lateral, and by coronal approach. Ophthalmology 1990; $97: 636-41$

4 Kendall-Taylor P, Crombie AL, Stephenson AM, Hardwick M, Hall K. Intravenous methylprednisolone in the treatment of Graves' ophthalmolopathy. Br Med f 1988; 297: 1574-8.

5 Harnett AN, Doughty D, Hirst A, Plowman PN. Radiotherapy in benign orbital disease. II: Ophthalmic Graves' disease and orbital histiocytosis X Brf Ophthalmol 1988; 72: 289-92.

6 Petersen IA, Kriss JP, McDougall IR, Donaldson SS. Prognostic factors in the radiotherapy of Graves' ophthalmopathy. Int $\mathcal{F}$ Radiation Oncology Biol Phys 1990; 19: 259-64.

7 Mourits MPh, Koornneef L, Wiersinga WM, Prummell MF, Berghout A, van der Gaag R. Clinical criteria for the assessment of disease actuvity in Graves ophthalmopathy: a novel approach. Br f Ophthalmol 1989; 73: 639-44.

8 Potts M, Falcao-Reis F, Fells P, Buceti S, Arden GB. Colour contrast sensitivity, pattern ERGs and cortical evoked potentials in dysthyroid optic neuropathy. Invest Ophthalmol Vis Sci 1990; 31: ARVO abstract 932.

9 Lyons CJ, Vickers SF, Lee JP. Botulinum toxin therapy in dysthyroid strabismus. Eye 1990; 4: 538-40.

10 Simonsz HJ, Kommerell G. In Graves' disease, increased muscle tension and reduced elasticity of affected muscles is primarily caused by active muscle contraction. Neuro-ophthalmology 1989; 9: 243-6. 